\title{
On New Extensions of Hilbert's Integral Inequality
}

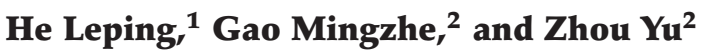 \\ ${ }^{1}$ Department of Mathematics and Applied Mathematics, College of Mathematics and Computer Science, \\ Jishou University, Jishou Hunan 416000, China \\ ${ }^{2}$ Department of Mathematics and Computer Science, Normal College of Jishou University, \\ Jishou Hunan 416000, China
}

Correspondence should be addressed to Gao Mingzhe, mingzhegao@163.com

Received 11 September 2007; Revised 5 November 2007; Accepted 11 December 2007

Recommended by Feng Qi

It is shown that some new extensions of Hilbert's integral inequality with parameter $\lambda(\lambda>1 / 2)$ can be established by introducing a proper weight function. In particular, when $\lambda=1$, a refinement of Hilbert's integral inequality is obtained. As applications, some new extensions of Widder's inequality and Hardy-Littlewood's inequality are given.

Copyright $(2008$ He Leping et al. This is an open access article distributed under the Creative Commons Attribution License, which permits unrestricted use, distribution, and reproduction in any medium, provided the original work is properly cited.

\section{Introduction and lemmas}

Let $f(x), g(x) \in L^{2}(0,+\infty)$. It is well known that the inequality of the form

$$
\iint_{\alpha}^{\infty} \frac{f(x) g(y)}{x+y-2 \alpha} d x d y \leq \pi\left\{\int_{\alpha}^{\infty} f^{2}(x) d x\right\}^{1 / 2}\left\{\int_{\alpha}^{\infty} g^{2}(x) d x\right\}^{1 / 2}
$$

is called Hilbert's integral inequality, where the coefficient $\pi$ is the best possible.

In [1], by introducing a parameter $\lambda(\lambda>1 / 2)$, the following extension of (1.1) of the form

$$
\iint_{0}^{\infty} \frac{f(x) g(y)}{x^{\lambda}+y^{\lambda}} d x d y \leq\left(\frac{\pi}{\lambda \sin (\pi / 2 \lambda)}\right)\left\{\int_{0}^{\infty} x^{1-\lambda} f^{2}(x) d x\right\}^{1 / 2}\left\{\int_{0}^{\infty} x^{1-\lambda} g^{2}(x) d x\right\}^{1 / 2}
$$

was established.

Recently, various improvements and extensions of (1.1) appear in a great deal of papers (see [2]). The aim of this paper is to give some new improvements of (1.1) and (1.2) and then present some important applications. 
We now introduce some notations that will be used throughout the paper.

Let $x-\alpha \geq 0, y-\alpha \geq 0, \lambda>1 / 2$, and let $c(x-\alpha)$ be an integrable function in $[\alpha,+\infty)$. Define $E(x, y)=1-c(x-\alpha)+c(y-\alpha)$ such that $E(x, y) \geq 0$ for $(x, y) \in(\alpha,+\infty) \times(\alpha,+\infty)$. We also define

$$
\begin{aligned}
& J_{1}=\iint_{\alpha}^{\infty} \frac{f^{2}(x)}{(x-\alpha)^{\curlywedge}+(y-\alpha)^{\lambda}}\left(\frac{x-\alpha}{y-\alpha}\right)^{1 / 2} E(x, y) d x d y, \\
& J_{2}=\iint_{\alpha}^{\infty} \frac{f^{2}(y)}{(x-\alpha)^{\lambda}+(y-\alpha)^{\lambda}}\left(\frac{y-\alpha}{x-\alpha}\right)^{1 / 2} E(x, y) d x d y .
\end{aligned}
$$

Lemma 1.1. With the above-mentioned assumptions, one has

$$
J_{1} J_{2}=\left(\frac{\pi}{\lambda \sin (\pi / 2 \lambda)}\right)^{2}\left\{\left(\int_{\alpha}^{\infty}(x-\alpha)^{1-\lambda} f^{2}(x) d x\right)^{2}-\left(\int_{\alpha}^{\infty} k(x) f^{2}(x) d x\right)^{2}\right\},
$$

where the weight function $k(x)$ is defined by

$$
k(x)=(x-\alpha)^{1-\lambda}\left\{\left(\frac{\lambda \sin (\pi / 2 \lambda)}{\pi}\right) \int_{0}^{\infty} \frac{c((x-\alpha) t) t^{-1 / 2}}{1+t^{\lambda}} d t-c(x-\alpha)\right\} .
$$

Proof. By using the substitution $t=(y-\alpha) /(x-\alpha)$, it is easy to deduce that

$$
\begin{aligned}
J_{1} & =\int_{\alpha}^{\infty}\left\{\int_{\alpha}^{\infty} \frac{1}{(x-\alpha)^{\lambda}\left(1+((y-\alpha) /(x-\alpha))^{\lambda}\right)}\left(\frac{x-\alpha}{y-\alpha}\right)^{1 / 2} E(x, y) d y\right\} f^{2}(x) d x \\
& =\int_{\alpha}^{\infty}\left\{\int_{0}^{\infty} \frac{1}{1+t^{\lambda}}\left(\frac{1}{t}\right)^{1 / 2}(1-c(x-\alpha)+c((x-\alpha) t)) d t\right\}(x-\alpha)^{1-\lambda} f^{2}(x) d x \\
& =\int_{\alpha}^{\infty}\left\{\frac{\pi}{\lambda \sin (\pi / 2 \lambda)}+\int_{0}^{\infty} \frac{c((x-\alpha) t)}{1+t^{\lambda}}\left(\frac{1}{t}\right)^{1 / 2} d t-\left(\frac{\pi}{\lambda \sin (\pi / 2 \lambda)}\right) c(x-\alpha)\right\}(x-\alpha)^{1-\lambda} f^{2}(x) d x \\
& =\left(\frac{\pi}{\lambda \sin (\pi / 2 \lambda)}\right)\left\{\int_{\alpha}^{\infty}(x-\alpha)^{1-\lambda} f^{2}(x) d x+\int_{\alpha}^{\infty} k(x) f^{2}(x) d x\right\},
\end{aligned}
$$

where $k(x)$ is a function defined by (1.5).

Similarly, we have

$$
J_{2}=\left(\frac{\pi}{\lambda \sin (\pi / 2 \lambda)}\right)\left\{\int_{\alpha}^{\infty}(x-\alpha)^{1-\lambda} f^{2}(x) d x-\int_{\alpha}^{\infty} k(x) f^{2}(x) d x\right\} .
$$

From the above equations involving $J_{1}$ and $J_{2},(1.4)$ holds true.

Lemma 1.2. Let $x-\alpha \geq 0$ and $\lambda>1 / 2$. Then

$$
\int_{0}^{\infty} \frac{t^{-1 / 2}}{\left(1+t^{\lambda}\right)\left(1+(x-\alpha)^{\lambda} t^{\lambda}\right)} d t= \begin{cases}\left(\frac{\pi}{\lambda \sin (\pi / 2 \lambda)}\right) \frac{(x-\alpha)^{\lambda-1 / 2}-1}{(x-\alpha)^{\lambda}-1}, & x-\alpha \neq 1, \\ \left(\frac{\pi}{\lambda \sin (\pi / 2 \lambda)}\right)\left(1-\frac{1}{2 \lambda}\right), & x-\alpha=1 .\end{cases}
$$


He Leping et al.

Proof. The case $x-\alpha \neq 1$ was studied in [3], or can be obtained by using [4]. Next, consider the case $x-\alpha=1$. By the definition and properties of beta function, it is easy to deduce that

$$
\int_{0}^{\infty} \frac{t^{-1 / 2}}{\left(1+t^{\lambda}\right)^{2}} d t=\frac{1}{\lambda} B\left(\frac{1}{2 \lambda}, 2-\frac{1}{2 \lambda}\right)=\frac{1}{\lambda}\left(1-\frac{1}{2 \lambda}\right) B\left(\frac{1}{2 \lambda}, 1-\frac{1}{2 \lambda}\right)=\left(1-\frac{1}{2 \lambda}\right) \frac{\pi}{\lambda \sin (\pi / 2 \lambda)} .
$$

\section{Theorem and its corollary}

Theorem 2.1. Let $f(x)$ and $g(x)$ be two real functions such that $0<\int_{\alpha}^{\infty}(x-\alpha)^{1-\lambda} f^{2}(x) d x<+\infty$ and $0<\int_{\alpha}^{\infty}(x-\alpha)^{1-\lambda} g^{2}(x) d x<+\infty$, where $\lambda>1 / 2$. Then

$$
\begin{aligned}
\left(\iint_{\alpha}^{\infty} \frac{f(x) g(y)}{(x-\alpha)^{\lambda}+(y-\alpha)^{\lambda}} d x d y\right)^{4} \\
\leq\left(\frac{\pi}{\lambda \sin (\pi / 2 \lambda)}\right)^{4}\left\{\left(\int_{\alpha}^{\infty}(x-\alpha)^{1-\lambda} f^{2}(x) d x\right)^{2}-\left(\int_{\alpha}^{\infty} \omega_{\lambda}(x) f^{2}(x) d x\right)^{2}\right\} \\
\quad \times\left\{\left(\int_{\alpha}^{\infty}(x-\alpha)^{1-\lambda} g^{2}(x) d x\right)^{2}-\left(\int_{\alpha}^{\infty} \omega_{\lambda}(x) g^{2}(x) d x\right)^{2}\right\}
\end{aligned}
$$

where the weight function $\omega_{\lambda}(x)$ is defined by

$$
\omega_{\lambda}(x)= \begin{cases}(x-\alpha)^{1-\lambda}\left\{\frac{(x-\alpha)^{\lambda-1 / 2}-1}{(x-\alpha)^{\lambda}-1}-\frac{1}{1+(x-\alpha)^{\lambda}}\right\}, & x-\alpha \neq 1 \\ \frac{1}{2}-\frac{1}{2 \lambda^{\prime}}, & x-\alpha=1 .\end{cases}
$$

Proof. First, assume $f=g$. Let $F(x, y)=f(x) f(y) /\left((x-\alpha)^{\lambda}+(y-\alpha)^{\lambda}\right), E(x, y)=1-c(x-\alpha)+$ $c(y-\alpha)$.

Then the following holds:

$$
\iint_{\alpha}^{\infty} F(x, y) d x d y=\iint_{\alpha}^{\infty} F(x, y) E(x, y) d x d y .
$$

In fact, it is obvious that

$$
\begin{aligned}
& \iint_{\alpha}^{\infty} F(x, y) E(x, y) d x d y \\
& \quad=\iint_{\alpha}^{\infty} F(x, y) d x d y-\iint_{\alpha}^{\infty} F(x, y) c(x-\alpha) d x d y+\iint_{\alpha}^{\infty} F(x, y) c(y-\alpha) d x d y .
\end{aligned}
$$

We need only to show that

$$
\iint_{\alpha}^{\infty} F(x, y) c(x-\alpha) d x d y=\iint_{\alpha}^{\infty} F(x, y) c(y-\alpha) d x d y .
$$


Let $\varphi(x)=\int_{\alpha}^{\infty} f(t) /\left((x-\alpha)^{\curlywedge}+(t-\alpha)^{\curlywedge}\right) d t$. Then

$$
\begin{aligned}
\iint_{\alpha}^{\infty} & F(x, y) c(x-\alpha) d x d y \\
& =\int_{\alpha}^{\infty}\left(\int_{\alpha}^{\infty} \frac{f(y)}{(x-\alpha)^{\lambda}+(y-\alpha)^{\lambda}} d y\right) f(x) c(x-\alpha) d x \\
& =\int_{\alpha}^{\infty}\left(\int_{\alpha}^{\infty} \frac{f(t)}{(x-\alpha)^{\lambda}+(t-\alpha)^{\lambda}} d t\right) f(x) c(x-\alpha) d x=\int_{\alpha}^{\infty} \varphi(x) f(x) c(x-\alpha) d x \\
& =\int_{\alpha}^{\infty} \varphi(y) f(y) c(y-\alpha) d y=\int_{\alpha}^{\infty}\left(\int_{\alpha}^{\infty} \frac{f(t)}{(y-\alpha)^{\lambda}+(t-\alpha)^{\lambda}} d t\right) f(y) c(y-\alpha) d y \\
& =\int_{\alpha}^{\infty}\left(\int_{\alpha}^{\infty} \frac{f(x)}{(y-\alpha)^{\lambda}+(x-\alpha)^{\lambda}} d x\right) f(y) c(y-\alpha) d y=\iint_{\alpha}^{\infty} F(x, y) c(y-\alpha) d x d y .
\end{aligned}
$$

Noting that $E(x, y) \geq 0$ and applying Schwarz's inequality, we have

$$
\begin{aligned}
\left(\iint_{\alpha}^{\infty} F(x, y) d x d y\right)^{2}= & \left(\iint_{\alpha}^{\infty} F(x, y) E(x, y) d x d y\right)^{2} \\
= & \left(\iint_{\alpha}^{\infty}\left\{\frac{f(x)}{\left((x-\alpha)^{\curlywedge}+(y-\alpha)^{\curlywedge}\right)^{1 / 2}}\left(\frac{x-\alpha}{y-\alpha}\right)^{1 / 4}(E(x, y))^{1 / 2}\right\}\right. \\
& \left.\times\left\{\frac{f(y)}{\left((x-\alpha)^{\lambda}+(y-\alpha)^{\lambda}\right)^{1 / 2}}\left(\frac{y-\alpha}{x-\alpha}\right)^{1 / 4}(E(x, y))^{1 / 2}\right\} d x d y\right)^{2} \\
\leq & \iint_{\alpha}^{\infty}\left\{\frac{f^{2}(x)}{(x-\alpha)^{\lambda}+(y-\alpha)^{\lambda}}\left(\frac{x-\alpha}{y-\alpha}\right)^{1 / 2} E(x, y)\right\} d x d y \\
& \times \iint_{\alpha}^{\infty}\left\{\frac{f^{2}(y)}{(x-\alpha)^{\lambda}+(y-\alpha)^{\lambda}}\left(\frac{y-\alpha}{x-\alpha}\right)^{1 / 2} E(x, y)\right\} d x d y=J_{1} J_{2}
\end{aligned}
$$

It follows from (1.4) that

$$
\left(\iint_{\alpha}^{\infty} F(x, y) d x d y\right)^{2} \leq\left(\frac{\pi}{\lambda \sin (\pi / 2 \lambda)}\right)^{2}\left\{\left(\int_{\alpha}^{\infty}(x-\alpha)^{1-\lambda} f^{2}(x) d x\right)^{2}-\left(\int_{\alpha}^{\infty} k(x) f^{2}(x) d x\right)^{2}\right\}
$$

where the weight function $k(x)$ is defined by (1.5).

Let $c(x)=1 /\left(1+x^{\lambda}\right)$, where $x \geq \alpha$ and $\lambda>1 / 2$. It is obvious that $E(x, y) \geq 0$. By Lemma 1.2 , it is easy to deduce that

$$
\begin{aligned}
k(x) & =(x-\alpha)^{1-\lambda}\left\{\left(\frac{\lambda \sin (\pi / 2 \lambda)}{\pi}\right) \int_{0}^{\infty} \frac{c((x-\alpha) t) t^{-1 / 2}}{1+t^{\lambda}} d t-c(x-\alpha)\right\} \\
& =(x-\alpha)^{1-\lambda}\left\{\left(\frac{\lambda \sin (\pi / 2 \lambda)}{\pi}\right) \int_{0}^{\infty} \frac{t^{-1 / 2}}{\left(1+t^{\lambda}\right)\left(1+(x-\alpha)^{\lambda} t^{\lambda}\right)} d t-c(x-\alpha)\right\}=\omega_{\lambda}(x) .
\end{aligned}
$$


He Leping et al.

Substitute $k(x)=\omega_{\lambda}(x)$ into $(2.8)$ to obtain

$$
\left(\iint_{\alpha}^{\infty} F(x, y) d x d y\right)^{2} \leq\left(\frac{\pi}{\lambda \sin (\pi / 2 \lambda)}\right)^{2}\left\{\left(\int_{\alpha}^{\infty}(x-\alpha)^{1-\lambda} f^{2}(x) d x\right)^{2}-\left(\int_{\alpha}^{\infty} \omega_{\lambda}(x) f^{2}(x) d x\right)^{2}\right\} .
$$

Next, consider the case $f \neq g$. By Schwarz's inequality, we have

$$
\begin{aligned}
& \left(\iint_{\alpha}^{\infty} \frac{f(x) g(y)}{(x-\alpha)^{\lambda}+(y-\alpha)^{\lambda}} d x d y\right)^{4} \\
& \quad=\left\{\left(\int_{0}^{1}\left(\int_{\alpha}^{\infty} t^{(x-\alpha)^{\lambda}-1 / 2} f(x) d x \int_{\alpha}^{\infty} t^{(y-\alpha)^{\lambda}-1 / 2} g(y) d y\right) d t\right)^{2}\right\}^{2} \\
& \quad \leq\left\{\int_{0}^{1}\left(\int_{\alpha}^{\infty} t^{(x-\alpha)^{\lambda}-1 / 2} f(x) d x\right)^{2} d t\right\}^{2}\left\{\int_{0}^{1}\left(\int_{\alpha}^{\infty} t^{(y-\alpha)^{\lambda}-1 / 2} g(y) d y\right)^{2} d t\right\}^{2} \\
& \quad=\left\{\iint_{\alpha}^{\infty} \frac{f(x) f(y)}{(x-\alpha)^{\lambda}+(y-\alpha)^{\lambda}} d x d y\right\}^{2}\left\{\iint_{\alpha}^{\infty} \frac{g(x) g(y)}{(x-\alpha)^{\lambda}+(y-\alpha)^{\lambda}} d x d y\right\}^{2} .
\end{aligned}
$$

Based on (2.10), it follows from (2.11) that the inequality (2.1) is valid at once. Theorem is proved.

The special case $\lambda=1$ in Theorem 2.1 yields the following Hilbert's integral inequality.

Corollary 2.2. If $0<\int_{\alpha}^{\infty} f^{2}(x) d x<+\infty$ and $0<\int_{\alpha}^{\infty} g^{2}(x) d x<+\infty$, then

$$
\begin{aligned}
\left(\iint_{\alpha}^{\infty} \frac{f(x) g(y)}{x+y-2 \alpha} d x d y\right)^{4} \leq & \pi^{4}\left\{\left(\int_{\alpha}^{\infty} f^{2}(x) d x\right)^{2}-\left(\int_{\alpha}^{\infty} \omega_{1}(x) f^{2}(x) d x\right)^{2}\right\} \\
& \times\left\{\left(\int_{\alpha}^{\infty} g^{2}(x) d x\right)^{2}-\left(\int_{\alpha}^{\infty} \omega_{1}(x) g^{2}(x) d x\right)^{2}\right\}
\end{aligned}
$$

where the weight function $\omega_{1}(x)$ is defined by

$$
\omega_{1}(x)=\frac{1}{\sqrt{x-\alpha}+1}-\frac{1}{x-\alpha+1} .
$$

Proof. It follows directly from the proof of Theorem 2.1 and so the details are omitted.

Remark 2.3. By setting $f=g$ in Corollary 2.2, (2.12) yields

$$
\left(\iint_{\alpha}^{\infty} \frac{f(x) f(y)}{x+y-2 \alpha} d x d y\right)^{2} \leq \pi^{2}\left\{\left(\int_{\alpha}^{\infty} f^{2}(x) d x\right)^{2}-\left(\int_{\alpha}^{\infty} \omega_{1}(x) f^{2}(x) d x\right)^{2}\right\}
$$

where the weight function $\omega_{1}(x)$ is defined by (2.13). 
Remark 2.4. For the case $\lambda=2$ in Theorem 2.1, (2.1) becomes

$$
\begin{aligned}
\left(\iint_{\alpha}^{\infty}\right. & \left.\frac{f(x) g(y)}{(x-\alpha)^{2}+(y-\alpha)^{2}} d x d y\right)^{4} \\
\leq & \frac{\pi^{4}}{4}\left\{\left(\int_{\alpha}^{\infty}(x-\alpha)^{-1} f^{2}(x) d x\right)^{2}-\left(\int_{\alpha}^{\infty} \omega_{2}(x) f^{2}(x) d x\right)^{2}\right\} \\
& \times\left\{\left(\int_{\alpha}^{\infty}(x-\alpha)^{-1} g^{2}(x) d x\right)^{2}-\left(\int_{\alpha}^{\infty} \omega_{2}(x) g^{2}(x) d x\right)^{2}\right\}
\end{aligned}
$$

where the weight function $\omega_{2}(x)$ is defined by

$$
\omega_{2}(x)= \begin{cases}(x-\alpha)^{-1}\left\{\frac{(x-\alpha)^{3 / 2}-1}{(x-\alpha)^{2}-1}-\frac{1}{1+(x-\alpha)^{2}}\right\}, & x-\alpha \neq 1 \\ \frac{1}{4}, & x-\alpha=1\end{cases}
$$

\section{Some applications}

As applications, we will give some extensions and refinements of Widder's inequality and Hardy-Littlewood's inequality.

Let $a_{n} \geq 0(n=0,1,2, \ldots), A(x)=\sum_{n=0}^{\infty} a_{n} x^{n}, A^{*}(x)=\sum_{n=0}^{\infty} a_{n} x^{n} / n !$. Then

$$
\int_{0}^{1} A^{2}(x) d x \leq \pi \int_{0}^{\infty}\left(e^{-x} A^{*}(x)\right)^{2} d x
$$

Inequality (3.1) is called Widder's inequality (see [5]).

We will give an extension of (3.1) below.

Theorem 3.1. Under the above assumptions, if $f(x)=e^{-(x-\alpha)} A^{*}(x-\alpha)$, then

$$
\left(\int_{0}^{1} A^{2}(x) d x\right)^{2} \leq \pi^{2}\left\{\left(\int_{\alpha}^{\infty} f^{2}(x) d x\right)^{2}-\left(\int_{\alpha}^{\infty} \omega_{1}(x) f^{2}(x) d x\right)^{2}\right\}
$$

where $\omega_{1}(x)$ is defined by (2.13).

Proof. First, observe that the following holds:

$$
\int_{0}^{\infty} e^{-t} A^{*}(t x) d t=\int_{0}^{\infty} e^{-t} \sum_{n=0}^{\infty} \frac{a_{n}(x t)^{n}}{n !} d t=\sum_{n=0}^{\infty} \frac{a_{n} x^{n}}{n !} \int_{0}^{\infty} t^{n} e^{-t} d t=\sum_{n=0}^{\infty} a_{n} x^{n}=A(x) .
$$

Let $t x=s-\alpha$. Then we have

$$
\begin{aligned}
\int_{0}^{1} A^{2}(x) d x & =\int_{0}^{1}\left\{\int_{0}^{\infty} e^{-t} A^{*}(t x) d t\right\}^{2} d x=\int_{0}^{1}\left(\int_{\alpha}^{\infty} e^{-(s-\alpha) / x} A^{*}(s-\alpha) d s\right)^{2} \frac{1}{x^{2}} d x \\
& =\int_{1}^{\infty}\left(\int_{\alpha}^{\infty} e^{-(s-\alpha) y} A^{*}(s-\alpha) d s\right)^{2} d y=\int_{0}^{\infty}\left(\int_{\alpha}^{\infty} e^{-(s-\alpha) u-(s-\alpha)} A^{*}(s-\alpha) d s\right)^{2} d u \\
& =\int_{0}^{\infty}\left(\int_{\alpha}^{\infty} e^{-(s-\alpha) u} f(s) d s\right)^{2} d u=\iint_{\alpha}^{\infty} \frac{f(s) f(t)}{s+t-2 \alpha} d s d t
\end{aligned}
$$


He Leping et al.

where $f(x)=e^{-(x-\alpha)} A^{*}(x-\alpha)$. Using Remark 2.3, the inequality (3.2) follows from (3.4) at once.

In particular, when $\alpha=0$, we obtain a refinement of (3.1).

Corollary 3.2. With the assumptions as Theorem 3.1, if $f(x)=e^{-x} A^{*}(x)$, then

$$
\left(\int_{0}^{1} A^{2}(x) d x\right)^{2} \leq \pi^{2}\left\{\left(\int_{0}^{\infty} f^{2}(x) d x\right)^{2}-\left(\int_{0}^{\infty} \omega_{1}(x) f^{2}(x) d x\right)^{2}\right\},
$$

where $\omega_{1}(x)$ is defined by (2.13).

Let $f(x) \in L^{2}(0,1)$. If $a_{n}=\int_{0}^{1} x^{n} f(x) d x, n=0,1,2, \ldots$, then we have Hardy-Littlewood's inequality (see [6]) of the form

$$
\sum_{n=0}^{\infty} a_{n}^{2} \leq \pi \int_{0}^{1} f^{2}(x) d x
$$

where $\pi$ is the best constant that keeps (3.6) valid. In [7], the inequality (3.6) was extended to the following inequality:

$$
\int_{0}^{\infty} f^{2}(x) d x \leq \pi \int_{0}^{1} h^{2}(x) d x
$$

where $f(x)=\int_{0}^{1} t^{x} h(x) d x, x \in[0,+\infty)$.

The inequality (3.7) is called Hardy-Littlewood's integral inequality. Afterwards, the inequality (3.7) was refined into the following form (see [8]):

$$
\int_{0}^{\infty} f^{2}(x) d x \leq \pi \int_{0}^{1} t h^{2}(t) d t
$$

We will give a new extension of (3.8) here.

Theorem 3.3. Let $\lambda>1 / 2, h(t) \in L^{2}(0,1)$, and $h(t) \neq 0$. Define a function by $f(x)=$ $\int_{0}^{1} t^{(x-\alpha)^{\lambda}}|h(t)| d t$. If $0<\int_{\alpha}^{+\infty}(x-\alpha)^{1-\lambda} f^{2}(x) d x<+\infty$, then

$$
\begin{aligned}
& \left(\int_{\alpha}^{\infty} f^{2}(x) d x\right)^{4} \\
& \quad \leq\left(\frac{\pi}{\lambda \sin (\pi / 2 \lambda)}\right)^{2}\left\{\left(\int_{\alpha}^{\infty}(x-\alpha)^{1-\lambda} f^{2}(x) d x\right)^{2}-\left(\int_{\alpha}^{\infty} \omega_{\lambda}(x) f^{2}(x) d x\right)^{2}\right\}\left(\int_{0}^{1} t h^{2}(t) d t\right)^{2},
\end{aligned}
$$

where the weight function $\omega_{\lambda}(x)$ is defined by (2.2).

Proof. By writing $f^{2}(x)$ in the form

$$
f^{2}(x)=\int_{0}^{1} f(x) t^{(x-\alpha)^{\lambda}}|h(t)| d t
$$


and applying Schwarz's inequality, we obtain

$$
\begin{aligned}
\left(\int_{\alpha}^{+\infty} f^{2}(x) d x\right)^{4} & =\left\{\int_{\alpha}^{\infty}\left(\int_{0}^{1} f(x) t^{(x-\alpha)^{\lambda}}|h(t)| d t\right) d x\right\}^{4} \\
& =\left\{\int_{0}^{1}\left(\int_{\alpha}^{+\infty} f(x) t^{(x-\alpha)^{\lambda}-1 / 2} d x\right) t^{1 / 2}|h(t)| d t\right\}^{4} \\
& \leq\left\{\int_{0}^{1}\left(\int_{\alpha}^{+\infty} f(x) t^{(x-\alpha)^{\lambda}-1 / 2} d x\right)^{2} d t \int_{0}^{1} t h^{2}(t) d t\right\}^{2} \\
& =\left\{\int_{0}^{1}\left(\iint_{\alpha}^{+\infty} f(x) f(y) t^{(x-\alpha)^{\lambda}+(y-\alpha)^{\lambda}-1} d x d y\right) d t \int_{0}^{1} t h^{2}(t) d t\right\}^{2} \\
& =\left(\iint_{\alpha}^{+\infty} \frac{f(x) f(y)}{(x-\alpha)^{\lambda}+(y-\alpha)^{\lambda}} d x d y\right)^{2}\left(\int_{0}^{1} t h^{2}(t) d t\right)^{2} .
\end{aligned}
$$

By (2.10), the inequality (3.9) follows from (3.11) at once.

Remark 3.4. By setting $\lambda=1$ and $\alpha=0$, we obtain the following refinement of (3.8):

$$
\left(\int_{0}^{\infty} f^{2}(x) d x\right)^{4} \leq \pi^{2}\left\{\left(\int_{0}^{\infty} f^{2}(x) d x\right)^{2}-\left(\int_{0}^{\infty} \omega_{1}(x) f^{2}(x) d x\right)^{2}\right\}\left(\int_{0}^{1} t h^{2}(t) d t\right)^{2},
$$

where the weight function $\omega_{1}(x)$ is defined by (2.13).

\section{Acknowledgments}

Authors would like to express their thanks to the referees for their valuable suggestions and comments. The research is supported by the Scientific Research Fund of Hunan Provincial Education Department, Grants no. 07C520 and 06C657.

\section{References}

[1] K. Jichang, "Note on new extensions of Hilbert's integral inequality," Journal of Mathematical Analysis and Applications, vol. 235, no. 2, pp. 608-614, 1999.

[2] M. Gao and L. C. Hsu, "A survey of various refinements and generalizations of Hilbert's inequalities," Journal of Mathematical Research E Exposition, vol. 25, no. 2, pp. 227-243, 2005.

[3] I. S. Gradshteyn and I. M. Ryzhik, Table of Integrals, Series, and Products, Academic Press, San Diego, Calif, USA, 2000.

[4] Y. Jin, Applied Integral Tables, Chinese Science and Technology University Press, Hefei, China, 2006.

[5] O. V. Widder, "An inequality related to Hilbert's inequalities," Journal of the London Mathematical Society, vol. 4, pp. 194-198, 1924.

[6] G. H. Hardy, J. E. Littlewood, and G. Pólya, Inequalities, Cambridge University Press, Cambridge, UK, 2nd edition, 1952.

[7] M. Gao, "On Hilbert's inequality and its applications," Journal of Mathematical Analysis and Applications, vol. 212, no. 1, pp. 316-323, 1997.

[8] M. Gao, T. Li, and L. Debnath, "Some improvements on Hilbert's integral inequality," Journal of Mathematical Analysis and Applications, vol. 229, no. 2, pp. 682-689, 1999. 


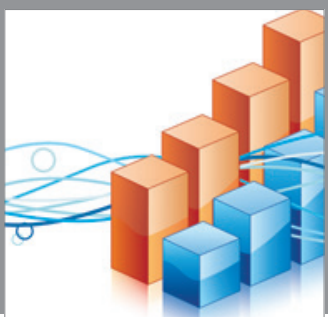

Advances in

Operations Research

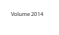

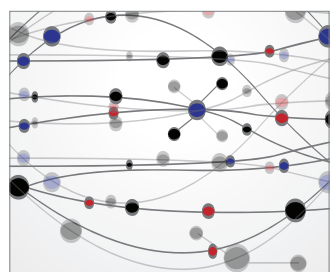

\section{The Scientific} World Journal
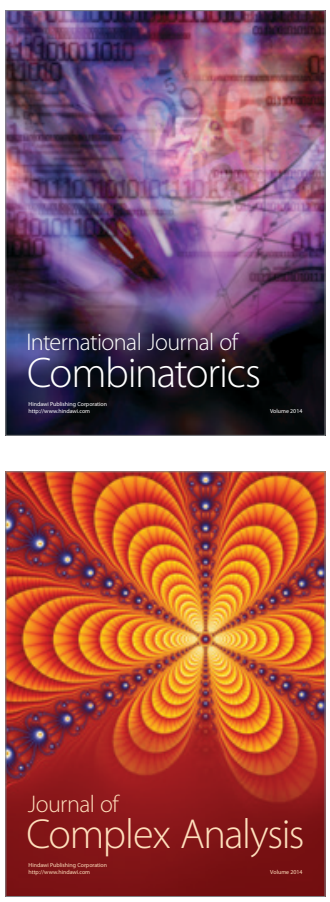

International Journal of

Mathematics and

Mathematical

Sciences
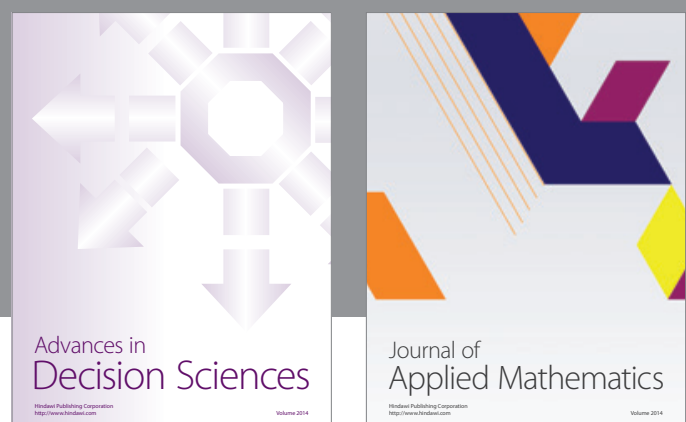

Journal of

Applied Mathematics
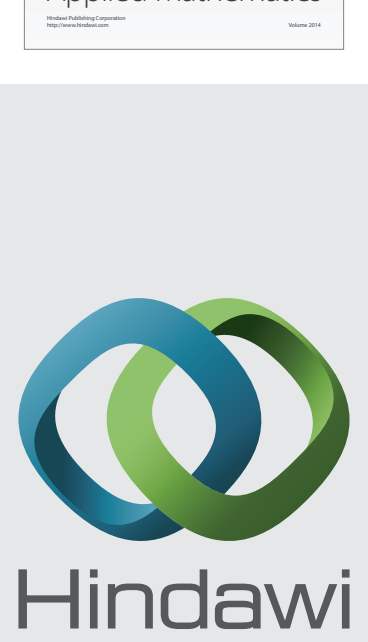

Submit your manuscripts at http://www.hindawi.com
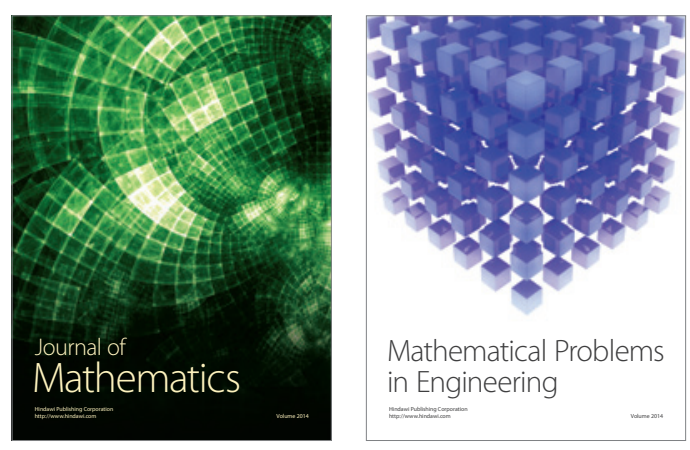

Mathematical Problems in Engineering
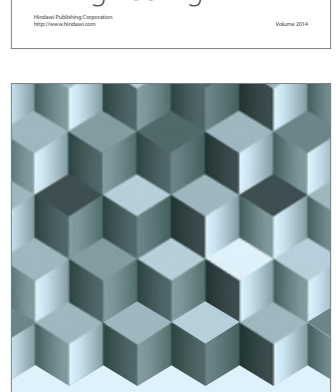

Journal of

Function Spaces
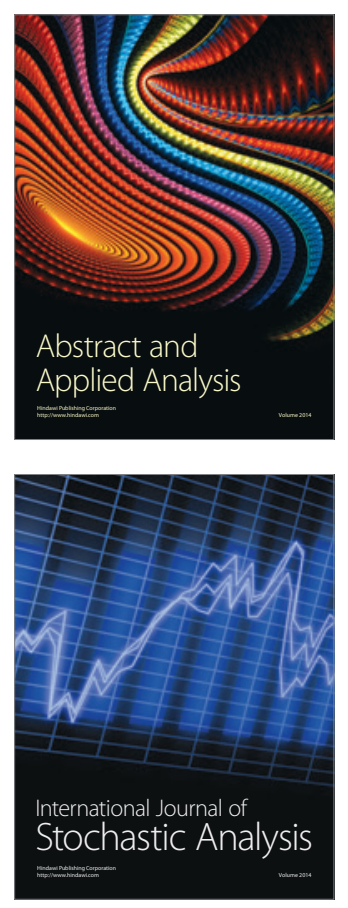

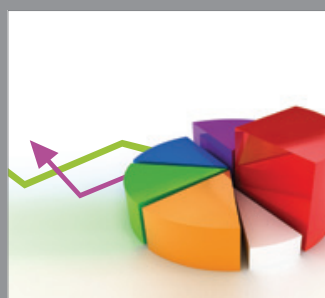

ournal of

Probability and Statistics

Promensencen
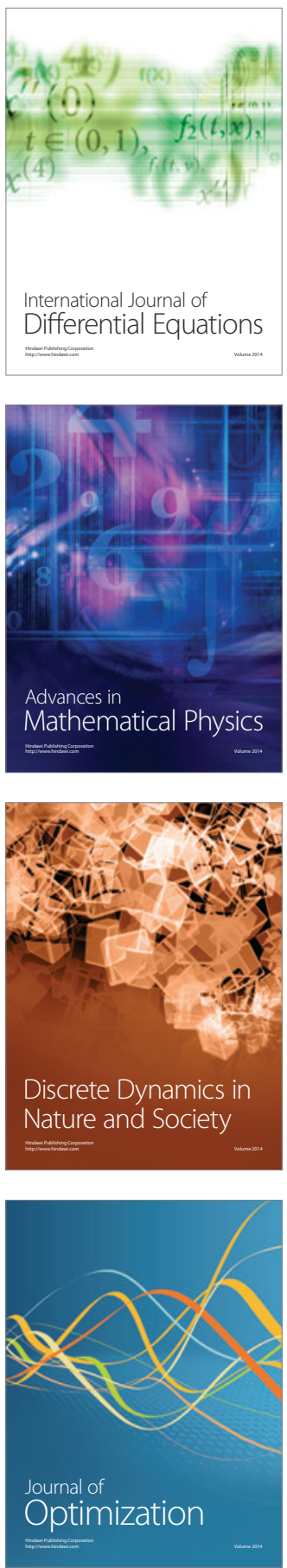